\title{
Complicações Neurológicas Determinadas pela Anestesia Subaracnóidea *
}

\section{Spinal Anesthesia-Induced Neurological Complications}

Eliana Marisa Ganem, TSA ${ }^{1}$, Yara Marcondes Machado Castiglia, TSA ${ }^{2}$, Pedro Thadeu Galvão Vianna, TSA ${ }^{2}$

\author{
RESUMO \\ Ganem EM, Castiglia YMM, Vianna PTG - Complicações Neu- \\ rológicas Determinadas pela Anestesia Subaracnóidea
}

Justificativa e Objetivos - Complicações neurológicas da anestesia subaracnóidea, apesar de raras, podem determinar seqüelas importantes. O objetivo deste estudo é apresentar estas complicações com a finalidade de esclarecer os fatores desencadeantes, o que facilita o diagnóstico das lesões.

Conteúdo - São apresentadas as seguintes complicações: lesão de nervo desencadeada pela agulha e cateter, cefaléia pós-punção, síndrome da artéria espinhal anterior, hematoma espinhal, meningite bacteriana, meningite asséptica, aracnoidite adesiva, síndrome da cauda eqüina e sintomas neurológicos transitórios.

Conclusões - O conhecimento dos fatores desencadeantes de complicações neurológicas determinadas pela anestesia subaracnóidea pode prevenir as lesões, diagnosticar e tratar mais precocemente e, desse modo, mudar o prognóstico das mesmas.

UNITERMOS: COMPLICAÇÕES: neurológicas; TÉCNICAS ANESTÉTICAS, Regional: subaracnóidea

\section{INTRODUÇÃO}

A s complicações neurológicas desencadeadas pela anestesia subaracnóidea são raras. Estudos publicados antes da década de 70, com levantamento totalizando mais de 50.000 anestesias raquidianas, mostraram que a incidência de alterações neurológicas sensitivas, como adormecimentos ou parestesias, variou de $0 \%$ a $0,7 \%$, enquanto que os déficits motores variaram de $0,005 \%$ a $0,02 \%{ }^{1-3}$.

Estudo prospectivo realizado na França constatou que a incidência de complicações neurológicas relacionadas à anestesia subaracnóidea foi de 6/10.000 anestesias ${ }^{4}$. Os principais fatores etiológicos desencadeantes de lesões foram o trauma ocasionado pela agulha de punção e a neurotoxicidade do anestésico local. De 40.640 anestesias raquidianas, observaram-se 5 casos de síndrome de cauda eqüina

\footnotetext{
* Recebido do (Received from) CET/SBA do Departamento de Anestesiologia da Faculdade de Medicina de Botucatu (FMB - UNESP), Botucatu, $S P$

1. Professora Adjunta Livre Docente do CET/SBA da FMB - UNESP

2. Professor(a) Titular do CET/SBA da FMB - UNESP
}

Apresentado (Submitted) em 04 de outubro de 2001

Aceito (Accepted) para publicação em 12 de dezembro de 2001

Correspondência para (Mail to):

Dra. Eliana Marisa Ganem

Dept ${ }^{\circ}$ de Anestesiologia da FMB - UNESP

Distrito de Rubião Junior

18618-970 Botucatu, SP

(C) Sociedade Brasileira de Anestesiologia, 2002

\section{SUMMARY}

Ganem EM, Castiglia YMM, Vianna PTG - Spinal Anesthesia-Induced Neurological Complications

Background and Objectives - Although rare, spinal anesthesia-induced neurological complications may determine serious sequelae. This study aimed at presenting these complications in order to elucidate triggering factors and help in the diagnosis of such injuries.

Contents - The following complications are presented: needle and catheter-induced nerve injury, post dural puncture headache, anterior spinal artery syndrome, spinal hematoma, septic meningitis, aseptic meningitis, adhesive arachnoiditis, cauda equina syndrome, and transient neurological symptoms.

Conclusions - The understanding of triggering factors of spinal anesthesia-induced neurological complications may prevent injuries and help early diagnosis and treatment, therefore changing patients prognosis.

KEY WORDS: ANESTHETIC TECHNIQUES, Regional: spinal block; COMPLICATIONS: neurological

(1/8.128 bloqueios). Outro estudo mostrou que lesões neurológicas permanentes ocorreram em 1/2.834 anestesias subaracnóideas ${ }^{5}$.

Vários fatores estão relacionados à etiologia dessas neuropatias, como injeções intraneurais de anestésico local ${ }^{6}$, contaminação de seringas com detergentes e anti-sépticos ${ }^{7}$, oxidantes contidos na preparação comercial do anestésico local ${ }^{8}$ e altas concentrações de anestésico local sobre o tecido nervoso ${ }^{9}$.

Existem fatores de risco que contribuem para o aparecimento das alterações neurológicas, dentre os quais a isquemia da medula espinhal (secundária ao uso de vasoconstritores e à hipotensão arterial prolongada), a lesão das raízes nervosas durante a introdução da agulha de cateter, as infecções e a própria solução de anestésico local escolhida ${ }^{9-11}$. O objetivo deste trabalho foi descrever as possíveis complicações neurológicas desencadeadas pela anestesia subaracnóidea.

\section{LESÃO DE NERVO DESENCADEADA PELA AGULHA OU CATETER}

As lesões originárias do trauma desencadeado pela agulha ou pelo cateter raramente resultam em lesões neurológicas permanentes ou incapacitantes. Em estudo retrospectivo envolvendo 4.767 anestesias a presença de parestesia durante a introdução da agulha ocorreu em $6,3 \%$ dos pacientes. Neste estudo, 6 pacientes apresentaram parestesia pós-operatória persistente e, dentre eles, 4 tinham apresen- 
tado este sintoma durante a realização da punção, o que torna a presença de parestesia durante a introdução da agulha, possível fator de risco para o aparecimento de parestesias persistentes $^{12}$.

Em estudo envolvendo 40.640 anestesias, foi observado que $2 / 3$ dos pacientes que apresentaram complicações neurológicas queixaram-se de dor durante a introdução da aguIha ou injeção do anestésico local. Em todos os casos o déficit neurológico teve a mesma distribuição da parestesia referida durante a realização da anestesia ${ }^{4}$.

Sempre que a agulha se afasta da linha média, intencionalmente, como nas punções paramedianas, ou não, existe a possibilidade de trauma de raiz nervosa. A parestesia unilateral em território de um dermátomo é indicador clássico de introdução da agulha proximamente ao nervo. Estruturas vasculares também localizam-se lateralmente à medula e lesões nestes vasos podem determinar hematomas ou mesmo interrupção de fluxo sangüíneo na região ${ }^{13}$.

Recentemente foram descritos alguns casos de lesão do cone medular em pacientes submetidas à anestesia subaracnóidea. As pacientes referiram dor à inserção da agulha ou à injeção do anestésico local. Em todos os casos foi utilizada a agulha com o bisel em ponta de lápis (Whitacre) e a punção foi realizada nos espaços intervertebrais $L_{2}-L_{3}$ ou $L_{1}-L_{2}{ }^{14}$. Quando são utilizadas agulhas de ponta de lápis, faz-se necessária a introdução da mesma em maior extensão no espaço subaracnóideo para a obtenção do líquor.

A passagem ou a presença de um cateter constitui fonte adicional de trauma. Dripps ${ }^{15}$ observou que a incidência de parestesia aumentou de $7 \%$ nas raquianestesias com punção única para $37,9 \%$ nas técnicas contínuas. Aincidência de alterações neurológicas aumentou de $0,13 \%$ para $0,66 \%$. Também Poulakka e col. ${ }^{16}$ observaram o dobro de incidência de parestesia quando se utilizou a técnica contínua.

\section{CEFALÉIA POR PUNÇÃO}

Cefaléia é a complicação mais comum da raquianestesia, com incidência estimada inferior a $3 \%$. Pode ser acompanhada de rigidez de nuca em $57 \%$ dos casos, de dor nas costas em $35 \%$ dos casos, de náusea em $22 \%$ dos casos e, em menor freqüência, de sintomas relacionados a comprometimento de pares cranianos e de espasmos musculares localizados $^{17}$.

Postula-se que o mecanismo responsável pelo seu aparecimento seja a diminuição da pressão liquórica decorrente da perda de líquor do espaço subaracnóideo através de lesões determinadas pela agulha de punção ou pelo cateter. Se a perda de líquor for superior à produção do mesmo $(0,3$ $\left.\mathrm{ml} \cdot \mathrm{min}^{-1}\right)$, poderá haver tração das estruturas intracranianas sempre que o paciente assumir a posição ereta. O paciente apresentará dor proveniente de estruturas sensíveis à dor via nervo trigêmeo (região frontal), glossofaríngeo, vago e nervos cervicais (região occipital, pescoço e ombros).
Um segundo componente da cefaléia pós-punção subaracnóidea é a vasodilatação cerebral, reação secundária ao estiramento vascular ${ }^{18}$

São considerados pacientes de risco para o aparecimento de cefaléia pós-punção, os adultos jovens e as gestantes. $O$ pico máximo de incidência é entre 20 e 29 anos, declinando após os 50 anos. O calibre e o tipo de agulha são fatores importantes no aparecimento da cefaléia. Aagulha ponta de lápis, porque separa ao invés de cortar as fibras da duramáter, o que facilita o fechamento do orifício determinado pela punção, tem se mostrado fator primordial na redução da cefaléia pós-raquianestesia ${ }^{19}$. Alguns autores encontraram incidência similar desta complicação, em pacientes obstétricas, quando utilizaram agulhas atraumáticas (Whitacre) e traumáticas (Quincke), desde que de fino calibre ${ }^{20}$. Em gestantes, a incidência de cefaléia pós-punção quando se utilizou a agulha de Whitacre $27 \mathrm{G}$ foi de $0,4 \%{ }^{21}$. Entretanto, outros autores ${ }^{22}$, estudando 5050 pacientes não-obstétricas, observaram menor incidência de cefaléia pós-punção quando utilizaram agulhas com bisel em ponta de lápis e agulhas com pequeno calibre.

Também postula-se que as punções paramedianas apresentam risco menor de ocasionar cefaléia pós-punção ${ }^{23}$, porque provocam menor perda de líquido em decorrência de efeito valvular desencadeado pela superposição da duramáter com a aracnóide ${ }^{24}$. Contudo, nem todos os estudos confirmam esta teoria. Imbelloni e col. ${ }^{25}$ encontraram incidência similar de cefaléia após punção mediana e paramediana. A cefaléia pós-punção aparece entre o $5^{\circ}$ e $7^{\circ}$ dias após a punção dural (na maioria dos casos, no segundo dia) ${ }^{19}$. Em aproximadamente $50 \%$ dos casos desaparece, espontaneamente, em 5 dias, e em $90 \%$ dos casos, em 10 dias.

Em presença de cefaléia é importante excluir sinais de irritação meníngea, febre, fotofobia ou sintomas neurológicos localizados que não são consistentes com o quadro clínico de cefaléia pós-punção.

O tratamento pode ser conservador e inclui hidratação, analgésicos e cafeína. Também é realizado por meio da administração peridural de solução fisiológica, cujo índice de sucesso ou cura é em torno de $50 \%$, ou de tampão sangüíneo peridural, cuja incidência de cura é de $90 \%$. Em pacientes obstétricas que apresentaram cefaléia pós-punção a terapêutica com tampão sangüíneo peridural curou $98,3 \%$ dos $\operatorname{casos}^{26}$. Porque o sangue se difunde cefalicamente dentro do espaço peridural, é recomendado que a introdução do mesmo seja abaixo do local no qual foi realizada a punção da duramáter.

\section{SÍNDROME DA ARTÉRIA ESPINHAL ANTERIOR DA MEDULA}

O suprimento sangüíneo da medula espinhal é precário em virtude da grande distância entre os vasos radiculares. A hipotensão arterial ou a insuficiência vascular localizada, associada ou não à anestesia subaracnóidea, podem produzir isquemia medular, resultando em síndrome da artéria espinhal anterior. Esta caracteriza-se por paralisia flácida dos membros inferiores, de início súbito, com reflexos segmen- 
tares abolidos, que acomete principalmente idosos com história prévia de aterosclerose ${ }^{27}$. Postula-se que anestésicos locais contendo vasoconstritores podem determinar isquemia em pacientes com doença microvascular ${ }^{28}$, porém esta hipótese ainda não foi confirmada ${ }^{29}$.

\section{HEMATOMA ESPINHAL}

Aincidência real de alterações neurológicas decorrentes de complicações hemorrágicas associadas a bloqueio neuroaxial não é conhecida. A incidência estimada após anestesia subaracnóidea é inferior a 1 para 220.000 bloqueios ${ }^{30}$.

Anormalidades anatômicas da coluna vertebral e medula espinhal, alterações na hemostasia e dificuldade na realização da punção raquidiana são consideradas fatores de risco para o aparecimento de hematoma espinhal ${ }^{31}$

Vandermeulen e col. ${ }^{32}$ descreveram 61 casos de hematomas associados à anestesia peridural e à subaracnóidea. Destes, 68\% (42 pacientes) apresentavam anormalidades hemostáticas. A anestesia subaracnóidea foi fator desencadeante de hematoma em 15 pacientes.

Existem poucos dados referentes ao risco de hematomas em pacientes tomando anticoagulantes orais e a maioria deles é relacionada à anestesia peridural; entretanto, é de consenso que a anestesia neuroaxial não deva ser realizada em pacientes totalmente anticoagulados ${ }^{18,33}$.

A segurança de se realizar bloqueio neuroaxial em pacientes heparinizados no per-operatório foi descrita por Rao e El-Etr ${ }^{34}$. Estes autores utilizaram a técnica contínua de anestesia e heparinizaram os pacientes 60 minutos após a colocação do cateter, não observando nenhuma complicação neurológica.

Ruff e Dougherty ${ }^{35}$ relataram hematoma em $2 \%$ dos pacientes (7/342) que foram submetidos à punção lombar e heparinizados em seguida. Nestes casos, a punção foi traumática, a anticoagulação foi precoce (menos que 1 hora após a punção) e houve terapia concomitante com aspirina, que foram considerados fatores de risco no desenvolvimento do hematoma.

Com relação à heparina subcutânea (SC), alguns autores ${ }^{36}$ realizaram revisão da literatura e não encontraram hematomas em mais de 5.000 pacientes que estavam recebendo esse medicamento por essa via para tromboprofilaxia e foram submetidos à raquianestesia ou à anestesia peridural. Entretanto, a literatura relata 3 casos de hematoma que associam bloqueios neuroaxias à heparina subcutânea em baixas doses, sendo apenas um relacionado à anestesia subaracnóidea $^{18}$.

O risco de sangramento, após punção em pacientes que estão recebendo heparina subcutânea, pode ser reduzido retardando-se a injeção do fármaco para depois da realização da anestesia e pode estar aumentado em pacientes debilitados ou após terapia prolongada com o medicamento ${ }^{37}$.

Com relação à heparina de baixo peso, apesar de muitos estudos clínicos atestarem a segurança de sua utilização associada a bloqueios neuroaxiais, foram descritos 40 casos de hematomas espinhais em período de 5 anos, em pacientes recebendo droga profilaticamente no pré-operatório ${ }^{31}$. Destes, seis ocorreram após anestesia subaracnóidea, um após a técnica contínua e 5 após punção única. Destes cinco, dois pacientes receberam o anticoagulante no dia da cirurgia, em dois houve trauma durante a realização da punção e um estava recebendo terapia antiplaquetária associada à heparina. Estima-se que a incidência de hematoma espinhal associado à heparina de baixo peso seja de $1 / 40.800$ raquianestesias $^{38}$. Em pacientes que estão utilizando a droga, recomenda-se que a punção seja realizada pelo menos de 10 a 12 horas após a administração da dose de heparina e, naqueles que recebem doses elevadas ( $1 \mathrm{mg} \cdot \mathrm{kg}^{-1}$, a cada 12 horas), é necessário que se retarde a punção por 24 horas ${ }^{31}$.

Quanto às medicações antiplaquetárias, vários estudos demonstram ser segura a realização de bloqueio em pacientes que estejam recebendo estes fármacos ${ }^{39-41}$. Deve-se, entretanto, estar alerta para o risco de hematomas quando drogas antiplaquetárias estão associadas a outros medicamentos que alteram os demais componentes da coagulação, como anticoagulantes orais, heparina de baixo peso e heparina ${ }^{42}$.

\section{MENINGITE ASSÉPTICA}

É uma síndrome clínica constituída por febre alta, cefaléia, rigidez de nuca e fotofobia. Quando relacionada à anestesia subaracnóidea, tem início agudo, dentro de 24 horas após a punção, com curso benigno, autolimitado ${ }^{7}$, e com duração de até uma semana ${ }^{43}$. À punção lombar, o líquor apresenta-se turvo, pressão elevada, com aumento de células brancas à custa de polimorfonucleares, aumento da concentração de proteínas e glicose normal ${ }^{44}$. O diagnóstico é confirmado pela ausência de microorganismos à microscopia e cultura negativa $^{7}$.

A contaminação de seringas com detergentes utilizados para a sua lavagem ${ }^{7,43,45}$ é o mais conhecido fator desencadeante de meninge asséptica. Outras causas também foram aventadas, como introdução de pirógenos contidos na solução anestésica, nas agulhas e nas seringas, presença de sangue e de proteínas introduzidos no espaço subaracnóideo durante a punção lombar ${ }^{44,46}$, ação de conservantes contidos nos anestésicos e administração de ranitidina, carbamazepina e antibióticos no espaço subaracnóideo ${ }^{47}$.

\section{MENINGITE BACTERIANA}

É rara, porém constitui emergência médica e apresenta mortalidade em torno de $30 \%{ }^{47}$. Caracteriza-se por febre alta, cefaléia intensa, consciência alterada e sinais de meningismo ${ }^{18}$ e inicia-se dentro de 48 horas após o bloqueio. A terapia com antibiótico pode retardar o aparecimento dos sintomas ${ }^{47}$.

O líquor apresenta-se com leucocitose às custas de polimorfonucleares, glicose baixa $\left(\leq 30 \mathrm{mg} \cdot \mathrm{dl}^{-1}\right)$, proteínas elevadas $\left(\geq 150 \mathrm{mg} \cdot \mathrm{dl}^{-1}\right)$ e bactérias à microscopia e à cultura.

A fonte de infecção pode ser exógena, por meio de contaminação da pele (Stafilococcus) ou por germes contidos na oro- 
faringe (Streptococcus), ou endógena, secundária a infecções longe do local de punção.

Foram descritos quatro casos de meningite iatrogênica, por Streptococcus, em período de 4 anos, após anestesias raquidianas realizadas pelo mesmo anestesiologista ${ }^{48}$. Em todos os casos o médico apresentava faringite recorrente e realizou o bloqueio sem máscara facial.

\section{ARACNOIDITE ADESIVA}

É uma das complicações mais graves da anestesia subaracnóidea. Consiste na diminuição de força muscular e alteração de sensibilidade nos membros inferiores e no períneo, acompanhadas por alterações vesicais e intestinais ${ }^{29}$. O quadro inicia-se lentamente, alguns dias ou semanas após a realização do bloqueio, podendo levar à paraplegia completa e, em alguns casos, à morte ${ }^{29}$.

Existe reação proliferativa das leptomeninges, com obliteração do espaço subaracnóide $0^{29,49}$ em decorrência da formação de trabéculas, levando à deformidade das raízes nervosas ${ }^{51}$ e constrição da medula espinhal ${ }^{29}$. Por obstruir o fluxo normal de líquor, a pressão intracraniana pode se elevar; entretanto, é o comprometimento do suprimento sangüíneo da medula espinhal e das raízes nervosas o principal responsável pelas alterações neurológicas encontradas ${ }^{49}$.

Os principais fatores etiológicos são a adição, ao anestésico local, de substâncias com a finalidade de alterar a gravidade específica da solução, como álcool e acácia, a contaminação do material utilizado na anestesia com anti-sépticos e detergentes ${ }^{49,50}$ e a presença de antioxidantes (bissulfito) na solução ${ }^{8}$.

\section{SÍNDROME DA CAUDA EQÜINA}

A síndrome da cauda eqüina é resultante de alterações na função dos componentes das raízes que a constituem ${ }^{51}$. Caracteriza-se por disfunção vesical e intestinal, perda da sensibilidade em área de períneo e graus variáveis de fraqueza muscular nos membros inferiores, sintomas que se iniciam imediatamente após a reversão do bloqueio subaracnóideo, podendo ser permanentes ou apresentar regressão parcial, lenta e gradual em períodos que variam de semanas a meses ${ }^{29}$.

Dentre os fatores potencialmente capazes de desencadeá-la encontram-se o trauma direto ou indireto das raízes nervosas, a isquemia, a infecção e as reações neurológicas ${ }^{9}$.

Enfoque particular é atribuído aos efeitos neurotóxicos dos anestésicos locais, já descritos na primeira metade do século passado ${ }^{52-54}$, porque a síndrome da cauda eqüina reapareceu em 1991, agora ligada à raquianestesia com a técnica contínua, pela qual se administrou lidocaína a $5 \%$ em solução de glicose a $7,5 \%$, através de microcatéteres ${ }^{9,55}$. Nestes casos, as doses do anestésico local foram mais elevadas que aquelas usualmente empregadas com injeção única e tiveram por finalidade aumentar a extensão de um bloqueio predominantemente sacral ${ }^{9}$.
Anatureza focal do bloqueio pressupôs estar havendo distribuição não uniforme do anestésico local, que foi injetado através de microcatéteres. Estes, em decorrência da alta resistência ao fluxo imposto pelo seu pequeno lúmen, propiciam injeções lentas que comprometem a dispersão do anestésico local no líquor ${ }^{56,57}$. Quando a droga se deposita ao redor das raízes sacrais, há contato prolongado com o tecido nervoso e probabilidade de ocorrer lesão neurológica, que é dependente da concentração ${ }^{11,58,59}$.

Nos últimos anos, foram descritos casos de síndrome de cauda eqüina após administração de grandes volumes de anestésico local, que inicialmente deveriam ser injetados no espaço peridural e inadvertidamente foram introduzidos no espaço subaracnóideo ${ }^{60-62}$. Nestes casos, postulou-se que o contato prolongado do anestésico local com o nervo, as doses e as concentrações elevadas poderiam ter determinado déficits neurológicos permanentes ${ }^{60}$.

Em 1999 foram publicados cinco casos de síndrome da cauda eqüina, agora após injeção única da lidocaína hiperbárica a $5 \%$ em doses que não ultrapassaram as recomendadas, e um caso desta lesão neurológica que ocorreu após injeção repetida do anestésico local por falha da anestesia ${ }^{63}$. É de conhecimento, desde 1991, após publicação de Drasner ${ }^{64}$, que falhas no bloqueio ou bloqueios inadequados podem indicar má distribuição do anestésico local no líquor e que doses repetidas de anestésico local podem resultar em concentrações tóxicas ao tecido nervoso.

Com relação aos demais casos de síndrome de cauda eqüina, após injeção única de anestésico local, cuja etiologia da lesão foi atribuída à neurotoxicidade do anestésico, recomenda-se que ao se utilizar lidocaína em anestesia subaracnóidea não se ultrapasse a dose de $60 \mathrm{mg}$ e a concentração de $2 \%{ }^{65}$.

\section{SÍNTOMAS NEUROLÓGICOS TRANSITÓRIOS}

Descritos por Schneider e col. em $1993^{66}$, os sintomas neurológicos transitórios (SNT) caracterizam-se por dor nas costas, que se irradia para as nádegas e face dorso-lateral das pernas, bilateralmente, sendo restrita aos dermátomos $\mathrm{L}_{5}-\mathrm{S}_{1}$, às vezes acompanhada por disestesias.

Esta dor inicia-se nas primeiras 24 horas que seguem a regressão da anestesia subaracnóidea (tempo que pode variar de 1 a 20 horas) sempre após período livre de sintomas ${ }^{66,67}$. Ador, na maioria dos casos, é de moderada intensidade, com duração média de 2 a 3 dias. Os reflexos músculo-tendinosos e as funções vesical e intestinal permanecem normais. Apresenta incidência extremamente variável, dependendo do anestésico estudado e da posição em que o paciente permanece no per-operatório. A incidência é maior quando se utiliza a lidocaína ${ }^{68-72}$, quando comparada a outros anestésicoslocais, como bupivacaína ${ }^{69}$, prilocaína ${ }^{71,73}$ e procaína ${ }^{74}$. Pacientes que permanecem em posição de litotomia no per-operatório apresentam incidência de SNT de 30 a 36\% $68,70,71$, pacientes que permanecem com o joelho flexionado para a realização de artroscopia apresentam incidência en- 
tre 18 e $22 \%{ }^{69,72,75,76}$ e pacientes em posição supina, entre 4 e $8 \%{ }^{69,74}$.

As possíveis causas de SNT incluem toxicidade do anestésico local ${ }^{65,77}$, isquemia neural secundária ao estiramento do nervo ciático ${ }^{66}$, acúmulo de AL secundário ao uso de agulhas de pequeno calibre ${ }^{78}$, espasmo muscular, pontos de gatilho miofaciais ${ }^{79}$, mobilização precoce e irritação nos gânglios das raízes dorsais ${ }^{80}$.

Em vista de que poucos pacientes, submetidos à raquianestesia com bupivacaína, apresentaram sintomas neurológicos transitórios, é pouco provável que os sintomas sejam decorrentes do bloqueio subaracnóideo ${ }^{76}$.

Sabe-se que a baricidade, a osmolaridade da solução, a adição de vasoconstritor e a concentração do anestésico local não são fatores determinantes para o aparecimento dos SNT $1,69,81$, assim como o tipo e o calibre da agulha ${ }^{82}$.

Em estudo prospectivo multicêntrico envolvendo 1863 pacientes, observou-se que a lidocaína, a posição de litotomia e o paciente ambulatorial são fatores que prevêem para o aparecimento de SNT ${ }^{82}$.

Como os sintomas neurológicos transitórios foram observados em vigência de uso de lidocaína em concentrações tão baixas quanto $0,5 \%{ }^{81}$, infere-se que os mecanismos responsáveis pelo seu aparecimento sejam diferentes daqueles que desencadeiam síndrome da cauda eqüina, ou seja, altas concentrações de anestésico local ${ }^{11}$.

A natureza radicular bilateral da dor pode sugerir neurotoxicidade específica da lidocaína, porém o período livre de sintomas entre a regressão do bloqueio e o aparecimento da dor e a falta de sinais objetivos consistentes de neurotoxicidade fala contra neurotoxicidade ${ }^{83}$.

Atualmente acredita-se que a lidocaína, porque determina maior relaxamento muscular, propicia estiramento músculo-esquelético quando o paciente permanece em posição de litotomia no per-operatório ${ }^{71,84}$.

Em estudo recente, com o objetivo de se determinar se os SNT eram resultado de neurotoxicidade direta da lidocaína, foram avaliados os pacientes que apresentavam SNT com auxílio de eletroneuromiografia, de estudos de condução nervosa e de potenciais evocados somatossensoriais e não foram observadas quaisquer alterações eletrofisiológicas em nenhum paciente, nem mesmo em áreas susceptíveis aos efeitos tóxicos dos anestésicos locais, como as raízes nervosas posteriores ${ }^{85}$.

No que diz respeito a SNT, a prevenção da síndrome é importante. Portanto, deve-se evitar a utilização da lidocaína em pacientes submetidos a anestesias ambulatoriais e naqueles em posição de litotomia.

\section{CONCLUSÕES}

As complicações neurológicas determinadas pela anestesia subaracnóidea são raras, entretanto podem ser devastadoras. A seleção adequada dos pacientes a serem submetidos à técnica, durante a avaliação pré-anestésica, pode preveni-las. A realização adequada da técnica anestésica tanto com relação à punção quanto à escolha do anestésico local pode diminuir a incidência e a avaliação pós-anestésica pode diagnosticar, possibilitando tratamento precoce e meIhorando o prognóstico dessas complicações.

\section{Spinal Anesthesia-Induced Neurological Complications}

Eliana Marisa Ganem, TSA, M.D., Yara Marcondes Machado Castiglia, TSA, M.D., Pedro Thadeu Galvão Vianna, TSA, M.D.

\section{INTRODUCTION}

Spinal anesthesia-induced neurological complications are rare events. Studies published before the 70's, involving more than 50,000 spinal anesthesias, have shown that the incidence of sensory neurological complications, such as dumbness or paresthesia, has varied from $0 \%$ to $0.7 \%$, while motor deficits have varied from $0.005 \%$ to $0.02 \%{ }^{1-3}$.

A French prospective study has observed that the incidence of neurological complications related to spinal anesthesia was 6:10,000 anesthesias ${ }^{4}$. Major etiologic triggering factors were needle or puncture trauma and local anesthetic neurotoxicity. There were 5 cauda equina syndrome cases out of 40,640 spinal anesthesias ( $1: 8,128$ blockades). Adifferent study has shown one permanent neurological injury out of $1: 2,834$ spinal anesthesias ${ }^{5}$.

Several factors are involved in the etiology of such neuropathies, such as intraneural injections of local anesthetics ${ }^{6}$, syringe contamination with detergents and anti-septic solutions ${ }^{7}$, oxidants contained in local anesthetics commercial preparations ${ }^{8}$ and high local anesthetic concentrations on nervous tissues ${ }^{9}$.

There are also risk factors associated to neurological changes, such as bone marrow ischemia (secondary to vasoconstrictors and prolonged arterial hypotension), nerve roots injury during needle and catheter introduction, infections and the local anesthetic solution per se ${ }^{9-11}$.

This study aimed at describing possible spinal anesthesia-induced neurological complications.

\section{NEEDLE OR CATHETER-INDUCED NERVE INJURY}

Injuries triggered by needle or catheter introduction seldom result in permanent or disabling neurological injuries. In a retrospective study involving 4,767 anesthetic procedures, there has been a $6.3 \%$ incidence of paresthesia during needle introduction. In this study, 6 patients had persistent postoperative paresthesia and, among them, 4 had presented the symptom during puncture, leading to the hypothesis that paresthesia during needle introduction was the risk factor for persistent paresthesias ${ }^{12}$.

In a study involving 40,640 anesthetic procedures, $2 / 3$ of patients with neurological complications had complained of 
pain during needle introduction or local anesthetic injection. In all cases, the neurological deficit had the same distribution of paresthesias referred during anesthetic induction ${ }^{4}$.

Whenever the needle is intentionally (paramedian punctures) or not apart from the midline there is the possibility of nerve root trauma. Unilateral paresthesia in the territory of one dermatome is a classic indicator of needle introduction close to the nerve. There are vascular structures laterally to the bone marrow and injuries to those vessels may cause hematomas or even blood flow interruption in the region ${ }^{13}$.

Some conus medullaris injuries in patients submitted to spinal anesthesia have been recently described. Patients referred pain at needle insertion or local anesthetic injection. In all cases, pencil point bevel needles were used (Whitacre) and puncture was performed in $L_{2}-L_{3}$ or $L_{1}-L_{2}$ interspace ${ }^{14}$. When pencil point needles are used, it is necessary to further introduce it in the spinal space to obtain cerebral spinal fluid (CSF).

The introduction or the presence of a catheter is an additional trauma source. Dripps ${ }^{15}$ has observed that the incidence of paresthesias increased from $7 \%$ in spinal anesthesias with single puncture to $37.9 \%$ in continuous techniques. The incidence of neurological complications increased from $0.13 \%$ to $0.66 \%$. Poulakka et al. ${ }^{16}$ have also observed twice the incidence of paresthesias with the continuous technique.

\section{POST DURAL PUNCTURE HEADACHE}

Headache is the most common spinal anesthesia complication, with an estimated incidence of less than $3 \%$. It may be followed by neck stiffness and, less frequently, by symptoms related to cranial nerves involvement and localized muscle spasms $^{17}$.

It is suggested that the triggering mechanism would be CSF pressure decrease caused by loss of spinal space CSF through injuries determined by the needle or the catheter. If CSF loss is higher than its production $\left(0.3 \mathrm{ml}_{\mathrm{min}}^{-1}\right)$ there might be intracranial structures traction whenever the patient stands up. Patients will present pain coming from pain-sensitive structures via trigeminal (frontal region), glossopharyngeal, vagus and cervical nerves (occipital region, neck and shoulders).

Asecond post dural puncture headache component would be brain vasodilatation secondary to vascular stretching ${ }^{18}$.

Young adults and pregnant women are patients at risk for post dural puncture headache. Maximum incidence is between 20 and 29 years of age, decreasing after 50 years of age. Needle size and type are major factors for headache. Pencil point needles, because they separate instead of cutting dural fibers, therefore helping puncture hole closing, are being a major factor in decreasing post dural puncture headache ${ }^{19}$. Some authors have found a similar incidence of this complication in obstetric patients with atraumatic (Whitacre) and traumatic (Quincke) needles, provided they were thin ${ }^{20}$. In pregnant women, the incidence of post dural puncture headache was $0.4 \%$ with $27 \mathrm{G}$ Whitacre needles ${ }^{21}$. Other authors ${ }^{22}$, however, studying 5050 non-obstetric patients, have ob- served a lower post dural puncture headache incidence when pencil point and thin needles were used.

It is also suggested that paramedian punctures have a lower risk for post dural puncture headache ${ }^{23}$ because they cause less SCF leakage due to the valvular effect triggered by the superimposition of dura and arachnoid ${ }^{24}$. However, not all studies have confirmed this theory. Imbelloni et al. ${ }^{25}$ have found a similar headache incidence after median and paramedian punctures.

Post dural puncture headache appears in the $5^{\text {th }}$ to $7^{\text {th }}$ day after puncture (more often in the $2^{\text {nd }}$ day) ${ }^{19}$. In approximately $50 \%$ of cases it spontaneously disappears in 5 days and in $90 \%$ of cases, in 10 days.

In the presence of headache, it is important to rule out meningeal irritation, fever, photophobia or localized neurological symptoms which are inconsistent with post dural puncture headache clinical presentation.

Treatment may be conservative and includes hydration, analgesics and caffeine. It may also be achieved by epidural saline solution, the healing rate of it is approximately $50 \%$, or by epidural blood patch, with a healing incidence of $90 \%$. In obstetric patients with post dural puncture headache, epidural blood patch has reverted $98.3 \%$ of cases ${ }^{26}$. Because the blood is cephalically spread within the epidural space, it is recommended to be introduced below the dural puncture site.

\section{ANTERIOR SPINAL CORD ARTERY SYNDROME}

Spinal cord blood supply is precarious, due to the long distance among radicular vessels. Arterial hypotension or localized vascular failure, associated or not to spinal anesthesia, may cause spinal cord ischemia, resulting in anterior spinal cord artery syndrome, characterized by sudden lower limb flaccid paralysis with abolished segmental reflexes and affecting especially elderly with previous history of atherosclerosis ${ }^{27}$. it is suggested that local anesthetics with vasoconstrictors may determine ischemia in microvascular disease patients ${ }^{28}$, but this hypothesis is still to be confirmed $^{29}$.

\section{SPINAL HEMATOMA}

The real incidence of neurological changes as a consequence of hemorrhagic complications associated to neuraxial block is not known, the post spinal anesthesia incidence estimated is less than 1 to 220,000 blockades ${ }^{30}$.

Spinal and back bone anatomic abnormalities, changes in hemostasis and difficult spinal puncture are considered risk factors for spinal hematomas ${ }^{31}$.

Vandermeulen et al. ${ }^{32}$ have described 61 hematomas associated to epidural and spinal anesthesia. From these, $68 \%$ (42 patients) had hemostatic abnormalities. Spinal anesthesia was the triggering factor for hematoma in 15 patients.

There are few data on the risk for hematoma in patients under oral anticoagulants and most of them are related to epidural anesthesia; it is a consensus, however, that neuraxial anes- 
thesia should not be induced in totally anticoagulated patients ${ }^{18,33}$.

Rao and El-Etr ${ }^{34}$ have described the safety of inducing neuraxial block in patients under perioperative heparin. These authors have used the continuous anesthesia technique and have administered heparin 60 minutes after catheter insertion without any neurological complication.

Ruff and Dougherty ${ }^{35}$ have reported hematoma in $2 \%$ of patients (7:342) submitted to lumbar punction followed by heparin. In these cases, puncture has been traumatic with early coagulation (less than 1 hour after puncture) and simultaneous aspirin therapy, considered risk factors for hematoma. As to subcutaneous heparin (SC), some authors ${ }^{36}$ have reviewed the literature and have not found hematomas in more than 5000 patients receiving this drug through this route for thromboprophylaxis and submitted to spinal or epidural anesthesia. However, the literature reports 3 cases of hematomas which associated neuraxial blocks to low subcutaneous heparin doses, being only one related to spinal anesthesia $^{18}$.

Post-puncture bleeding risk in patients receiving subcutaneous heparin may be decreased by administering the drug after anesthesia, and may be increased in weak patients or after prolonged heparin therapy ${ }^{37}$.

As to low molecular weight heparin, although many studies reporting the safety of its association to neuraxial blocks, 40 spinal hematomas in a 5-year period were reported in patients receiving prophylactic preoperative low molecular weight heparin ${ }^{31}$. From these, six appeared after spinal anesthesia, one after continuous technique and five after single puncture. From those five, two patients received anticoagulants in the day of the surgery, two suffered trauma during surgery and one was receiving anti-platelet therapy associated to heparin.

It is estimated that the incidence of spinal hematoma associated to low molecular weight heparin is $1: 40,800$ spinal anesthesias ${ }^{38}$. In patients using the drug, it is recommended that puncture be performed at least 10 to 12 hours after heparin administration and, in those receiving high doses (1 $\mathrm{mg} \cdot \mathrm{kg}^{-1}$ at every 12 hours), puncture should wait for 24 hours ${ }^{31}$.

Several studies have shown the safety of inducing blockade in patients under anti-platelet therapy ${ }^{39-41}$. Care must be taken, however, with the risk for hematomas when anti-platelet drugs are associated to drugs changing other coagulation components, such as oral anticoagulants, low molecular weight heparin and heparin ${ }^{42}$.

\section{ASEPTIC MENINGITIS}

It is a clinical syndrome characterized by high fever, headache, neck stiffness and photophobia. When related to spinal anesthesia it has a sudden onset within 24 hours after puncture with benign and self-limited evolution ${ }^{7}$ and lasting up to one week ${ }^{43}$. At lumbar puncture, CSF is turbid, with high pressure, increased white blood cells at the expenses of polymorphonuclears, increased protein concentration and normal glucose ${ }^{44}$. Diagnosis is confirmed by the absence of microorganisms at microscopy and negative culture ${ }^{7}$. Syringe contamination with detergents $7,43,45$ is the best known triggering factor for aseptic meningitis. Other causes were also considered, such as the introduction of pyrogens contained in the anesthetic solution, needles and syringes, blood and proteins introduced in the spinal space during lumbar puncture ${ }^{44,46}$, the action of anesthetic preservatives and the administration of ranitidin, carbamazepine and antibiotics in the spinal space ${ }^{47}$.

\section{SEPTIC MENINGITIS}

Although rare, it is a medical emergency with a mortality rate of approximately $30 \%{ }^{47}$. It is characterized by high fever, severe headache, changes in consciousness and signs of meningism ${ }^{18}$, and starts within 48 hours after blockade. Antibiotic therapy may delay the appearance of symptoms ${ }^{47}$.

CSF presents leucocytosis at the expenses of polymorphonuclears, low glucose $\left(\leq 30 \mathrm{mg}^{-\mathrm{dl}^{-1}}\right)$, high proteins $(\geq 150$ $\mathrm{mg} \cdot \mathrm{dl}^{-1}$ ) and bacteria at microscopy and culture.

Infection sources may be exogenous, through skin contamination (Staphylococcus) or oropharyngeal germs (Streptococcus), or endogenous secondary to infections distant from the puncture site.

Four iatrogenic Streptococcus meningitis cases in a 4-year period were described after spinal anesthesias performed by the same anesthesiologist ${ }^{48}$. In all cases, the physician had pharyngitis and induced blockade without facial mask.

\section{ADHESIVE ARACHNOIDITIS}

It is one of the most severe spinal anesthesia complications. It consist in the decrease in muscle strength and changes in lower limbs and perineal sensitivity, followed by vesical and intestinal changes ${ }^{29}$. Symptoms start slowly, some days or weeks after blockade and may lead to total paraplegia and, in some cases, to death ${ }^{29}$.

There is a leptomeningeal proliferative reaction, with spinal space obliteration ${ }^{29,49}$ as a consequence of trabeculae formation and leading to nerve root deformities ${ }^{51}$ and spinal cord constriction ${ }^{29}$. For obstructing normal CSF flow, intracranial pressure may increase; however, neuraxial and nerve roots blood flow impairment is the major responsible for the neurological changes ${ }^{49}$.

Major etiologic factors are the association to local anesthetics of substances aiming at changing solution's weight, such as alcohol and acacia, the contamination of anesthetic material with anti-septic solutions and detergents ${ }^{49,50}$ and the presence of antioxidants (bisulfite) in the solution ${ }^{8}$.

\section{CAUDA EQUINA SYNDROME}

Cauda equina syndrome is the consequence of changes in root components function ${ }^{51}$. It is characterized by vesical and intestinal dysfunction, loss of perineal sensitivity and variable degrees of lower limb muscle weakness, symptoms 
which initiate immediately after spinal blockade reversion and may be permanent or with partial, slow and gradual recovery in periods varying from weeks to months ${ }^{29}$.

Direct or indirect nerve root trauma, ischemia, infection and neurological reactions are among potential cauda equina syndrome triggering factors ${ }^{9}$.

Special attention is given to local anesthetics neurotoxic effects already described during the first half of last century $52-54$, because cauda equina syndrome has reappeared in 1991 , now related to continuous spinal anesthesia with $5 \%$ lidocaine in $7.5 \%$ glucose through microcatheters ${ }^{9,55}$. In those cases, local anesthetic doses were higher than those usually used in single injection and were aimed at increasing the extension of a predominantly sacral blockade ${ }^{9}$.

The focal nature of the blockade led to the hypothesis of a non-uniform local anesthetic spread, was been injected through microcatheters. These, due to the high flow resistance imposed by their narrow lumen, allow for slow injections affecting local anesthetic CSF spread ${ }^{56,57}$. When the drug is deposited around sacral roots, there is a prolonged contact with nervous tissues and the possibility of concentration-dependent neurological injuries ${ }^{11,58,59}$.

Cases of cauda equina syndrome have been recently described after the administration of high local anesthetic volumes which should be initially injected in the epidural space and were inadvertently introduced in the spinal space ${ }^{60-62}$. In these cases, the hypothesis was that the prolonged local anesthetic contact with the nerve, in high doses and concentrations could have determined permanent neurological deficits ${ }^{60}$

In 1999, five cases of cauda equina syndrome were reported after a single $5 \%$ hyperbaric lidocaine injection in recommended doses, and one case after repeated local anesthetic injections due to anesthesia failure ${ }^{63}$. It is known that, since 1991 , after a study by Drasner ${ }^{64}$, blockade failures or inadequate blockades may indicate a poor local anesthetic CSF spread and that repeated local anesthetic doses may result in toxic concentrations in nervous tissues.

As to other cauda equina syndrome cases after single local anesthetic injection with etiology attributed to anesthetic neurotoxicity, it is recommended that spinal lidocaine dose should not go beyond $60 \mathrm{mg}$ at a concentration of $2 \%{ }^{65}$.

\section{TRANSIENT NEUROLOGICAL SYMPTOMS}

Described by Schneider et al. ${ }^{66}$ in 1993, transient neurological symptoms (TNS) are characterized by back pain irradiating to the buttocks and legs dorsolateral face bilaterally, being restricted to dermatomes $L_{5}-S_{1}$, sometimes followed by dysesthesias.

Pain starts within 24 hours after spinal anesthesia recovery (varying from 1 to 20 hours) always after a period without symptoms ${ }^{66,67}$. In most cases, pain is moderate with a mean duration of 2 to 3 days. Tendon reflexes and vesical and intestinal functions remain normal. The incidence is extremely variable depending on the anesthetic and the perioperative po- sition of the patient. The incidence is higher with lidocaine 68-72, as compared to other local anesthetics, such as bupivacaine ${ }^{69}$, prilocaine ${ }^{71,73}$ and procaine ${ }^{74}$. Patients in perioperative lithotomy position have a $30 \%$ to $36 \%$ incidence of TNS $68,70,71$, patients remaining with bended knee for arthroscopy have an $18 \%$ to $22 \%$ incidence ${ }^{69,72,75,76}$, and patients in the supine position, between $4 \%$ and $8 \%{ }^{69,74}$.

Possible TNS causes are local anesthetic toxicity ${ }^{65,77}$, neural ischemia secondary to sciatic nerve stretching ${ }^{66}$, LA building up secondary to thin needles ${ }^{78}$, muscle spasm, myofacial triggering points ${ }^{79}$, early mobilization and dorsal root ganglia irritation ${ }^{80}$.

Since few patients submitted to spinal anesthesia with bupivacaine presented transient neurological symptoms, it is less probable that symptoms are a consequence of spinal block $^{76}$.

It is known that solution's baricity and osmolarity, the addition of vasconstrictors, local anesthetic concentration, needle type and size ${ }^{82}$ are not factors determining TNS ${ }^{1,69,81}$.

In a prospective multicentric study involving 1863 patients, it has been observed that lidocaine, lithotomy position and outpatient procedures are risk factors for TNS ${ }^{82}$.

Since transient neurological symptoms were observed with lidocaine in concentrations as low as $0.5 \%{ }^{81}$, the assumption is that mechanisms responsible for its appearance are different from those triggering cauda equina syndrome, that is, high local anesthetic concentrations ${ }^{11}$.

The bilateral radicular pain may suggest specific lidocaine neurotoxicity, but the period free of symptoms between blockade reversion and pain, and the lack of objective and consistent neurotoxicity signs talk against neurotoxicity ${ }^{83}$.

Currently, it is believed that lidocaine, for determining more muscle relaxation, allows for musculoskeletal stretching when patients remain in perioperative lithotomy position ${ }^{71,84}$. In a recent study aiming at determining whether TNS was a result of direct lidocaine neurotoxicity, TNS patients were evaluated by electromyography, nervous conduction and somatosensory evoked potential and no electrophysiological changes were found, not even in areas susceptible to local anesthetic toxic effects, such as posterior nerve roots ${ }^{85}$. It is important to prevent transient neurological syndrome symptoms. So, lidocaine should be avoided in patients submitted to outpatient anesthesia or in those in lithotomy position.

\section{CONCLUSIONS}

Spinal anesthesia-induced neurological complications are rare, however with devastating effects. The adequate preanesthetic selection of patients to be submitted to the technique may prevent them. The adequate technique, both in terms of puncture and local anesthetic choice, may decrease the incidence, and postanesthetic evaluation may diagnose them, allowing for early treatment and improving the prognosis of such complications. 


\section{REFERÊNCIAS - REFERENCES}

01 Dripps RD, Vandam LD - Long-term follow-up of patients who received 10.098 spinal anesthetics: failure to discover major neurological sequelae. JAMA, 1954;156:1486-1491.

02 Vandam LD, Dripps RD - Exacerbation of pre-existing neurologic disease after spinal anesthesia. N Engl J Med, 1956,255: 843-849.

03. Phillips OC, Ebner H, Nelson AT et al - Neurologic complications following spinal anesthesia with lidocaine: a prospective review of 10.440 cases. Anesthesiology, 1969;30:284-289.

04 Auroy Y, Narchi P, Messiah A et al - Serious complications related to regional anesthesia. Anesthesiology, 1997;87:479-486.

05 Dahegren N, Törnebrandt K - Neurological complications after anaesthesia. A follow-up of 18000 spinal and epidural anaesthetics performed over three years. Acta Anaesthesiol Scand, 1995;39:872-880.

06. Gentili F, Hudson AR, Hunter D et al - Nerve injection injury with local anesthetic agents: A light and electron microscopic, fluorescent microscopic, and horseradish peroxidase study. Neurosurgery 1980;6:263-272.

07. Bert AA, Laasberg LH - Aseptic meningitis following spinal anesthesia a complication of the past? Anesthesiology 1985;62: 674-677

08. Wang BC, Spielholz NI, Hillman DE et al - Subarachnoid sodium bisulfite (the antioxidant in nesacaine) causes chronic neurological deficit. Anesthesiology, 1982;57:A194

09. Rigler ML, Drasner K, Krejcie TC et al - Cauda equina syndrome after continuous spinal anesthesia. Anesth Analg, 1991;72: 275-281.

10. Myers RR., Sommer C - Methodology for spinal neurotoxicity studies. Reg Anesth, 1993;18: 439-447.

11. Ready LB, Plumer $\mathrm{MH}$, Haschke $\mathrm{RH}$ et al - Neurotoxicity of intrathecal local anesthetics in rabbits. Anesthesiology, 1985;63:364-370.

12. Horlocker TT, McGregor DG, Matsushige DK et al - A retrospective review of 4767 consecutive spinal anesthetics: central nervous system complications. Anesth Analg, 1997;84:578-584.

13. Herman $\mathrm{N}$ - Neurologic complication of regional anesthesia. Semin Anesth Perioper Med Pain, 1998;17:64-72.

14. Reynolds, F - Damage to the conus medullaris following spinal anaesthesia. Anaesthesia, 2001;56:235-247.

15. Dripps RD - A comparison of the malleable needle and catheter techniques for continuous spinal anesthesia. NY State J Med 1950;50:1595-1599.

16. Puolakka R, Haasio J, Pitkänen $M$ et al - Technical aspects and postoperative sequelae of spinal and epidural anesthesia: a prospective study of 3.230 orthopedic patients. Reg Anesth Pain Med 2000;25:488-497.

17. Neal MJ - Update on postdural puncture headache. Tech Reg Anesth Pain Manage, 1998;2:202-210.

18. Horlocker TT - Complications of spinal and epidural anesthesia. Anesthesiol Clin North Am, 2000;18:461-485.

19. Galinski S, Choi PT-L, Lucas S - The quality of PDPH literature in obstetrical anesthesia - results from an obstetrical PDPH bibliographic database. Anesthesiology, 1999;91:A1138.

20. Neves JFNP, Monteiro GA, Almeida JR et al - Raquianestesia para cesariana: avaliação da cefaléia com agulhas de Quincke e Whitacre 25G e 27G. Rev Bras Anestesiol, 1999;49:173-175.

21. Villar GCP, Rosa C, Cappelli EL et al - Incidência de cefaléia pós-raquianestesia em pacientes obstétricas com uso de agulha de Whitacre calibre 27G. Experiencia com 4570 casos. Rev Bras Anestesiol, 1999;49:110-112.

22. Imbelloni LE, Sobral MGC, Carneiro ANG - Cefaléia pós-raquianestesia e o desenho das agulhas. Experiência com 5050 casos. Rev Bras Anestesiol, 2001;51:43-52.

23. Halpern S, Preston R - Postdural puncture headache and spinal needle design - Metaanalyses. Anesthesiology, 1994;81: 1376-1383

24. Haffalvi BI - The dynamics of postspinal headache. Headache, 1977:17:64-67.

25. Imbelloni LE, Sobral MGC, Carneiro ANG - Influência do calibre da agulha, da via de inserção da agulha e do número de tentativas de punção na cefaléia pós-raquianestesia. Estudo prospectivo. Rev Bras Anestesiol, 1995;45:377-382.
26. Pedrosa GC, Jardim JL, Palmeira MA - Tampão sangüíneo peridural e alta hospitalar precoce: análise de 60 pacientes portadores de cefaléia pós-raquianestesia. Rev Bras Anestesiol, 1996;46:8-12.

27. Wedel DJ, Horlocker TT - Risks of regional anesthesia - infectious, septic. Reg Anesth, 1996; 21:57-61.

28. Kalichmam MW, Calcutt NA - Local anesthetic induced conduction block and nerve fiber injury in streptozotocin-diabetic rats. Anesthesiology, 1992; 77:941-947

29. Kane RE - Neurologic deficits following epidural or spinal anesthesia. Anesth Analg, 1981;60:150-161.

30. Tryba M - Epidural regional anesthesia and low molecular heparin: pro (German). Anästh Intensivmed Notfallmed Schmerzther, 1993; 28:179-181.

31. Horlocker TT, Wedel DJ - Neuraxial block and low molecular weight heparin: balancing perioperative analgesia and thromboprophylaxis. Reg Anesth Pain Med, 1998;23:164-177.

32. Vandermeulen EP, Van Aken $\mathrm{H}$, Vermylen $\mathrm{J}$ - Anticoagulants and spinal-epidural anesthesia. Anesth Analg, 1994;79:1165-1177.

33. Horlocker TT, Wedel DJ - Neurologic complications of spinal and epidural anesthesia. Reg Anesth Pain Med, 2000;25:83-98.

34. Rao TLK, El-Etr AA - Anticoagulation following placement of epidural and subarachnoid catheters: an evaluation of neurologic sequelae. Anesthesiology, 1981;55:618-620.

35. Ruff RL, Dougherty JH - Complications of lumbar puncture followed by anticoagulation. Stroke, 1981;12:879-881.

36. Schwander D, Bachmann F - Heparin and spinal or epidural anesthesia: decision analysis (review). Ann Fr Anesth Reanim, 1991;10: 284-296.

37. Liu SS, Mulroy MF - Neuroaxial anesthesia and analgesia in presence of standard heparin. Reg Anesth Pain Med, 1998;23: 157-163.

38. Schroeder DR - Statistic: detecting a rare adverse drug reaction using spontaneous reports. Reg Anesth Pain Med, 1998;23: 183-189.

39. Horlocker TT, Wedel DJ, Offord KP - Does preoperative antiplatelet therapy increase the risk of hemorrhagic complications associated with regional anesthesia? Anesth Analg, 1990;70:631-634.

40. CLASP (Collaborative low-dose aspirin study in pregnancy). CLASP: a randomized trial of low-dose aspirin for the prevention and treatment of pre-eclampsia among 9364 pregnant women. Lancet, 1994;343:619-629.

41. Horlocker TT, Wedel DJ, Schroeder DR et al - Preoperative antiplatelet therapy does not increase the risk of spinal hematoma associated with regional anesthesia. Anesth Analg, 1995;80: 303-309.

42. Urmey WF, Rowlingson JC - Do antiplatelet agents contribute to the development of perioperative spinal hematoma? Reg Anesth Pain Med, 1998;23:146-151.

43. Goldman WW, Sanford JP - An "Epidemic" of chemical meningitis. Am J Med 1960;29:94-101.

44. Harding SA, Collis RE, Morgan BM - Meningitis after combined spinal-extradural anaesthesia in obstetrics. $\mathrm{Br} \mathrm{J}$ Anaesth, 1994;73:545-547.

45. Rendell C - Chemical meningitis due to syringes stored in lysol. Anaesthesia, 1954;9:281-285

46. Phillips OC - Aseptic meningitis following spinal anesthesia. Anesth Analg, 1970;49:867-871.

47. Gorce $P$, Varlet $C$, Quaknine B et al - Méningites après anesthésie locorégionale rachidienne. Ann Fr Anesth Réanim, 2000;19: 375-381

48. Schneeberger PM, Janssen M, Voss A - Alpha hemolytic streptococci: a major pathogen of iatrogenic meningitis following lumbar puncture. Case reports and a review of the literature. Infection, 1996;24:29-33.

49. Greene NM - Neurological sequelae of spinal anesthesia. Anesthesiology, 1961;22:682-689.

50. Martelete $M$ - Sequelas neurológicas de anestesias peridurais. Relato de 4 casos. Rev Bras Anestesiol, 1981;31:245-250.

51. Jaradeh S - Cauda equina syndrome: a neurologist's perspective. Reg Anesth, 1993,18:473-480.

52. Sourasky M, Leeds MD - Overdose in spinal analgesia. Br Med J, 1926;18:524. 
53. Ferguson FR, Watkins $\mathrm{KH}$ - Paralysis of the bladder and associated neurological sequelae of spinal anaesthesia (cauda equina syndrome). Br J Surg, 1938;25:735-52.

54. Nicholson MJ, Eversole UH - Neurologic complications of spinal anesthesia. J Am Med Ass, 1946;132:679-85.

55. Schell RM, Brauer FS, Cole DJ et al - Persistent sacral nerve root deficits after continuous spinal anaesthesia. Can J Anaesth, 1991;38:908-911.

56. Rigler ML, Drasner K - Distribution of catheter-injected local anesthetic in a model of the subarachnoid space. Anesthesiology, 1991;75:684-692

57. Lambert DH, Hurley RJ - Cauda equina syndrome and continuous spinal anesthesia. Anesth Analg, 1991;72:817-819.

58. Vianna PTG, Vane LA, Yong LC et al - Alterações morfológicas da medula espinhal em cães submetidos à injeção hiperbárica de tetracaína a $1,2 \%$, lidocaína a $5 \%$ e glicose a $10 \%$. Rev Bras Anestesiol, 1985;35:(Suppl5):S1-S6.

59. Ganem EM, Vianna PTG, Marques M et al - Neurotoxicity of subarachnoid hyperbaric bupivacaine in dogs. Reg Anesth, 1996;21:234-238

60. Cheng AC - Intended epidural anesthesia as possible cause of cauda equina syndrome. Anesth Analg, 1994;78:157-159.

61. Drasner K, Rigler ML, Sessler DI et al - Cauda equina syndrome following intended epidural anesthesia. Anesthesiology, 1992;77:582-585

62. Lee DS, Bui T, Ferrarese J et al - Cauda equina syndrome after incidental total spinal anesthesia with $2 \%$ lidocaine. J Clin Anesth, 1998;10:66-69.

63. Loo CC, Irestedt L - Cauda equina syndrome after spinal anaesthesia with hyperbaric $5 \%$ lidocaine: a review of six cases of cauda equina syndrome reported to the Swedish pharmaceutical insurance 1993-1997. Acta Anaesthesiol Scand, 1999;43: 371-379.

64. Drasner K - Repeat injection after a "failed spinal"; at times, a potentially unsafe practice. Anesthesiology, 1991;75:713-714.

65. Drasner K - Lidocaine in spinal anesthesia: a vanishing therapeutic index? Anesthesiology, 1997;87:469-472.

66. Schneider M, Ettlin T, Kaufmann M et al - Transient neurologic toxicity after hyperbaric subarachnoid anesthesia with 5\% lidocaine. Anesth Analg, 1993;76:1154-1157

67. Sjöström S, Bläss J - Severe pain in both legs after spinal anaesthesia with hyperbaric 5\% lignocaine solution. Anaesthesia, 1994;49:700-702

68. Hampl KF, Schneider MC, Thorin D et al - Hyperosmolarity does not contribute to transient radicular irritation after spinal anesthesia with hyperbaric 5\% lidocaine. Reg Anesth, 1995;5: 363-368.

69. Pollock JE, Neal JM, Stephenson CA et al - Prospective study of the incidence of transient radicular irritation in patients undergoing spinal anesthesia. Anesthesiology, 1996;84:1361-1367.

70. Hampl KF, Schneider MC, Pargger H - A similar incidence of transient neurologic symptoms after spinal anesthesia with $2 \%$ and $5 \%$ lidocaine. Anesth Analg, 1996;83:1051-1054

71. Hampl KF, Heinzmann-Wiedmer S, Luginbuehl I et al - Transient neurologic symptoms after spinal anesthesia. A lower incidence with prilocaine and bupivacaine than with lidocaine. Anesthesiology, 1998;88:629-633

72. Hodgson PS, Liu SS, Batra MS et al - Procaine compared with lidocaine for incidence of transient neurologic symptoms. Reg Anesth Pain Med, 2000;25:218-222.

73. Weert K, Traksel M, Gielsen M et al - The incidence of transient neurological symptoms after spinal anaesthesia with lidocaine compared to prilocaine. Anaesthesia, 2000;55:1003-1024.
74. Martinez-Bourio R, Arzuaga M, Quintana JM et al - Incidence of transient neurologic symptoms after hyperbaric subarachnoid anesthesia with $5 \%$ lidocaine and $5 \%$ prilocaine. Anesthesiology, 1998;88:624-628

75. Liguori GA, Zayas VM, Chisholm MF - Transient neurologic symptoms after spinal anesthesia with mepivacaine and lidocaine. Anesthesiology, 1998;88:619-623.

76. Pollock JE - Management of the patient who develops transient neurologic symptoms after spinal anesthesia with lidocaine. Tech Reg Anesth Pain Manage, 2000;4:155-160.

77. Jong RH - Last round for a "heavy weight"? Anesth Analg, 1994;78: 3-4.

78. Beardesley D, Holman S, Gantt R et al - Transient neurologic deficit after spinal anesthesia: local anesthetic maldistribuition with pencil point needles? Anesth Analg, 1995;81:314-320.

79. Naveira FA, Copeland S, Anderson M et al - Transient neurologic toxicity after spinal anesthesia, or is it myofascial pain? Two case reports. Anesthesiology, 1998;88:268-270.

80. Dahlgren N - Transient radicular irritation after spinal anesthesia replay 2. Acta Anaesthesiol Scand, 1996;40:865.

81. Pollock JE, Liu SS, Neal JM et al - Dilution of spinal lidocaine does not alter the incidence of transient neurologic symptoms. Anesthesiology, 1999;90:445-450.

82. Freedman JM, Li DK, Drasner K et al - Transient neurologic symptoms after spinal anesthesia: an epidemiologic study of 1,863 patients. Anesthesiology, 1998; 89: 633-641.

83. Carpenter RL - Hyperbaric lidocaine spinal anaesthesia: do we need an alternative? Anesth Analg, 1995;81:1125-1128.

84. Pollock JE, deJong RH - Hyperbaric lidocaine for spinal anesthesia? Am J Anesthesiol, 1997;24:161-165.

85. Pollock JE, Burkhead D, Neal D - Spinal nerve function in five volunteers experiencing transient neurologic symptoms after lidocaine subarachnoid anesthesia. Anesth Analg, 2000;90: 658-665.

\section{RESUMEN}

Ganem EM, Castiglia YMM, Vianna PTG - Complicaciones Neurológicas Determinadas por la Anestesia Subaracnóidea

Justificativa y Objetivos - Complicaciones neurológicas de la anestesia subaracnóidea, a pesar de raras, pueden determinar secuelas importantes. El objetivo de este estudio es presentar estas complicaciones con la finalidad de esclarecer los factores desencadenantes, lo que facilita el diagnóstico de las lesiones.

Contenido - Son presentadas las siguientes complicaciones: lesión del nervio desencadenada por la aguja y catéter, cefalea pós-punción, síndrome de la arteria espinal anterior, hematoma espinal, meningitis bacteriana, meningitis aséptica, aracnoidite adhesiva, síndrome de la cola de caballo y síntomas neurológicos transitorios.

Conclusiones - El conocimiento de los factores desencadenantes de complicaciones neurológicas determinadas por la anestesia subaracnóidea puede prevenir las lesiones, diagnosticar y tratar más precozmente y, de ese modo, cambiar el pronóstico de las mismas. 